\title{
ON AN ALGORITHM OF G. BIRKHOFF \\ CONCERNING DOUBLY STOCHASTIC MATRICES
}

\author{
Diane M. Johnson, A.L. Dulmage and.N.S. Mendelsohn
}

(received June 20, 1960)

In [1] G. Birkhoff stated an algorithm for expressing a doubly stochastic matrix as an average of permutation matrices. In this note we prove two graphical lemmas and use these to find an upper bound for the number of permutation matrices which the Birkhoff algorithm inay use.

A doubly stochastic matrix is a matrix of non-negative elements with row and column sums equal to unity and is therefore a square matrix. A permutation matrix is an $n \times n$ doubly stochastic matrix which has $n^{2}-n$ zeros and consequently has $n$ ones, one in each row and one in each column. It has been shown by Birkhoff [1], Hoffman and Wielandt [5] and von Neumann [7] that the set of all doubly stochastic matrices, considered as a set of points in a space of $n^{2}$ dimensions constitute the convex hull of permutation matrices. In other words, any doubly stochastic matrix $A$ is expressible as a linear combination $c_{1} A_{1}+c_{2} A_{2}+\ldots+c_{t} A_{t}$ where the $A_{i}$ are permutation matrices and the $c_{i}$ are $\geqslant 0$ with $\sum c_{i}=1$. We say $A$ is an average of $A_{1}, A_{2}, \ldots, A_{t}$.

The graph of an $m \times n$ matrix $A=\left(a_{i j}\right)$ of non-negative elements is the bipartite graph $K$ for which one vertex set is the set $S=\left(s_{1}, s_{2}, \ldots, s_{m}\right)$ of rows and the other vertex set is the set $T=\left(t_{1}, t_{2}, \ldots, t_{n}\right)$ of columns, The unordered pair $\left[s_{i}, t_{j}\right]$ is an edge of $K$ if and only if $a_{i j}$ is greater than zero. The edges of $K$ corresponding to the places where a 1 appears in a permutation matrix will be called a diagonal of edges of $\mathrm{K}$.

Research supported in part by the United States Air Force Office of Scientific Research under Contract AF49(638)-860.

Canad. Math. Bull. vol. 3, no. 3, September 1960 
In [3] and [4] the structure and canonical decomposition of a bipartite graph have been discussed and an irreducible graph has been defined in [4].

LEMMA 1. A bipartite graph $K$ is the graph of some doubly stochastic matrix if and only if the canonical decomposition of $K$ is the disjoint union of irreducible subgraphs.

Proof. Let $A$ be an $n \times n$ doubly stochastic matrix and let $K$ be its bipartite graph. Since a doubly stochastic matrix is an average $A=c_{1} A_{1}+c_{2} A_{2}+\ldots+c_{t} A_{t}$ of permutation matrices, every non-zero element $a_{i j}=c_{1} \varepsilon_{1}+c_{2} \varepsilon_{2}+\ldots+c_{t} \varepsilon_{t}$ where $\varepsilon_{i}=1$ or 0 for all $i$ and there exists at least one integer $p, 1 \leqslant p \leqslant t$, such that $\varepsilon_{p}=1$. The presence of $c_{p} A_{p}$ in the expression for $A$ ensures that the edge $\left[s_{i}, t_{j}\right]$ belongs to a diagonal of edges in $K$. Thus every edge of $\bar{K}$ is admissible [3] and the graph of $K$ is identical with the first core of $\mathrm{K}$. In the canonical decomposition, as displayed in figure 1 of [4], there can be no rectangular blocks such as $\mathrm{X}_{\mathrm{i}} \times \mathrm{W}_{\mathrm{i}}$ or $\mathrm{U}_{\mathrm{i}} \times \mathrm{V}_{\mathrm{i}}$ for each such block contains a subgraph of $K$ which is the graph of a doubly stochastic submatrix of $A$ and every doubly stochastic matrix is square. Thus the canonical decomposition of $K$ consists of $k$ disjoint irreducible subgraphs $G_{i}$ with vertex sets $S_{i}$ and $\mathrm{T}_{\mathrm{i}}, \mathrm{i}=1,2,3, \ldots, \mathrm{k}$.

Conversely if $\mathrm{K}$ is the union of disjoint irreducible subgraphs, a doubly stochastic matrix $A$ for which $K$ is the graph can be constructed as follows. Let $N$ be the number of edges. There exists at least one diagonal containing each edge. Let $A_{i}$ be a permutation matrix the graph of which is a diagonal through the i th edge. The matrix

$$
A=\frac{1}{N} \sum_{i=1}^{n} A_{i}
$$

is a doubly stochastic matrix which has $K$ as graph.

In [3] the exterior dimension $E(K)$ of a bipartite graph $\mathrm{K}$ is defined.

LEMMA 2. Let $\mathrm{K}$ be an $\mathrm{n} \times \mathrm{n}$ bipartite graph which has a canonical decomposition consisting of $k$ disjoint irreducible subgraphs and let $K^{\prime}$ be any proper subgraph of $K$ which has a canonical decomposition consisting of $k^{\prime}\left(k^{\prime}>k\right)$ disjoint irreducible subgraphs with $E(K)=E\left(K^{\prime}\right)=n$. If $p$ is the 
number of blocks of the core of the decomposition which have the same vertex sets in $K$ and $K^{\prime}$, and if $N(K)$ and $N\left(K^{\prime}\right)$ are respectively the number of edges in $K$ and $K^{\prime}$ respectively, then $N(K)-N\left(K^{\prime}\right) \geqslant k^{\prime}-p$.

Proof. Using the notation of [4] section $3, K$ is the union of $k$ disjoint irreducible subgraphs $G_{i}=\left(S_{i} \times T_{i}\right) \cap K$ for $i=1,2, \ldots, k$. Let $N\left(G_{i}\right)=n_{i}, n_{1}+n_{2}+\ldots+n_{k}=N(K)$. $\mathrm{K}^{\prime}$ is the union of $\mathrm{k}^{\prime}$ disjoint irreducible subgraphs $G_{i}^{\prime}=\left(S^{\prime}{ }_{i} \times T^{\prime}{ }_{i}\right) \cap K^{\prime}$ for $i=1,2, \ldots, k^{\prime}$. Let $N\left(G_{i}^{\prime}\right)=n_{i}^{\prime}, n_{1}^{\prime}+n_{2}^{\prime}+\ldots+n_{k^{\prime}}^{\prime}=N\left(K^{\prime}\right)$. We may index the blocks of the core so that we have $G_{i}^{\prime} \subset G_{i}$ for $i=1,2, \ldots$, p. Thus $n_{i} \geqslant n_{i}^{\prime}$ for $i=1,2, \ldots$. There exist $q_{1}>1, q_{2}>1, \ldots, q_{k-p}>1$, where $q_{1}+q_{2}+\ldots+q_{k-p}=k^{\prime}-p$, such that the union of the $q_{1}$ disjoint irreducible subgraphs $G^{\prime} p+1, G^{\prime} p+2, \ldots, G^{\prime} p+q_{1}$ is a subgraph of $G_{p+1}$ and, in general, the union of the $\mathrm{qm}_{\mathrm{m}}$ disjoint irreducible subgraphs $G^{\prime} h_{m}+1{ }^{\prime} G^{\prime} h_{m}+2, \cdots, G^{\prime} h_{m}+q_{m}\left(h_{m}=q_{I}+q_{2}+\ldots+q_{m-1}\right)$ is a subgraph of $G_{p+m}, m=1,2, \ldots, k-p$.

Now consider the induced graph which results from the partitions $S_{p+1}=S^{\prime}{ }_{p+1} \cup S^{\prime}{ }_{p+2} \cup \ldots \cup S^{\prime}{ }_{p+q}{ }^{\prime}$ $T_{p+1}=T_{p+1}^{\prime} \cup T^{\prime}{ }_{p+2} \cup \ldots \cup T^{\prime}{ }_{p+q_{1}}, G_{p+1} \cdot$ Since $\left(S^{i} p+j \times T^{\prime} p+j\right) \cap G_{p+1}$ is irreducible for $j=1,2, \ldots, q_{1}$, the induced $q_{1} \times q_{1}$ bipartite graph $I$ is irreducible by [4] theorem 3 and hence has at least $2 q_{1}$ edges. Thus $I$ has at least $q_{1}$ edges in addition to $\left[S^{\prime}{ }_{p+j}, T^{\prime}{ }_{p+j}\right], j=1,2, \ldots, q_{1}$. It follows that $G_{p+1}$ has at least $q_{1}$ edges which are not edges of $G_{p+1}^{\prime} \cup G_{p+2}^{\prime} \cup \ldots \cup G_{p+q_{1}}^{\prime}$ Thus

$$
n_{p+1} \geqslant \sum_{j=1}^{q_{1}} n_{p+j}^{\prime}+q_{1}
$$

and, similarly,

$$
n_{p+m} \geqslant \sum_{j=1}^{q_{m}} n_{h_{m}}+j+q_{m}
$$

for $m=1,2, \ldots, k-p$. Thus

$$
N(K)=\sum_{i=1}^{p} n_{i}+\sum_{m=1}^{k-p} n_{p+m}
$$




$$
\begin{aligned}
& \geqslant \sum_{i=1}^{p} n_{i}+\sum_{r=1}^{k^{\prime}-p} n_{p+r}^{\prime}+\sum_{m=1}^{k-p} q_{m} \\
& =N\left(K^{\prime}\right)+k^{\prime}-p .
\end{aligned}
$$

COROLLARY. Since $p \leqslant k-1$, we have the weaker result

$$
N(K)-N\left(K^{\prime}\right) \geqslant\left(k^{\prime}-k\right)+1
$$

Lemma 2 may be generalised as follows. If $K$ is any $n \times n$ bipartite graph in which $E(K)=n$, and if $K^{\prime}$ is any subgraph of $K$ such that $E\left(K^{\prime}\right)=n$, then the induced bipartite graph I contains at least $q_{1}$ edges at least one of which is not in $G^{\prime}+1 \cup G^{\prime} p+2 \cup \ldots \cup G^{\prime}{ }_{p+q}$. The rest of these $q_{1}$ edges are inadmissible in $K^{\prime}$ and admissible in $K$. Accordingly, if $u$ is the total number of inadmissible edges of $K^{\prime}$ which are admissible in $K, N(K)-N\left(K^{\prime}\right)+u \geqslant k^{\prime}-p$.

Let $\mathrm{K}$ be an $\mathrm{n} \times \mathrm{n}$ bipartite graph which is the union of disjoint irreducible subgraphs. Let $t_{k}$ be the smallest integer such that every doubly stochastic matrix $A$ for which $K$ is the bipartite graph is expressible as an average of not more than $t_{k}$ permutation matrices. It was mentioned in [2] that the algorithm described by Birkhoff [1] never requires more than $n^{2}-n+1$ permutation matrices. It has been shown in [6], using a dimensional argument that $t_{k} \leqslant n^{2}-2 n+2$. However, this is an exis tence proof and does not show how to construct the average.

The main result of this note is the following.

THEOREM. Let $\mathrm{A}$ be an $\mathrm{n} \times \mathrm{n}$ doubly stochastic matrix with bipartite graph $K$. If $N$ is the number of edges in $K$ and $k$ is the number of disjoint irreducible subgraphs in the canonical decomposition of $K$, then $t_{k} \leq N-2 n+k+1$.

The Birkhoff algorithm expresses $A$ as an average of at most $t_{k}$ permutation matrices.

Proof. The Birkhoff construction is as follows. Let $c_{1}$ be the smallest (possibly unique) non-zero element of $A$. There exists a diagonal of non-zeros of which $c_{1}$ is the least. Let $A_{1}$ be the permutation matrix with ones on this diagonal, and let $A(1)=A-c_{1} A_{1}$. Repeating this process, let $A(2)=A(1)-c_{2} A_{2}$. Continuing, there must exist an integer $t$ such that $A(t-1)=$ $A(t-2)-c_{t-1} A_{t-1}$ and $A(t-1)$ has a diagonal of $n$ non-zero 
equal elements and $n^{2}-n$ zeros. The integer $t$ may vary depending on the choice of the diagonals. $A(t-1)=c_{t} A_{t}$ and $A=c_{1} A_{1}+c_{2} A_{2}+\ldots+c_{t} A_{t}$. Denote $A$ by $A(0)$ and let $K(i)$ be the graph of $A^{(i)}, i=0,1,2, \ldots, t-1$. We have $E(K(i))=n$ for $i=0,1,2, \ldots, t-1 . A(i)$ has equal row and column sums, and hence by lemma $1, K^{(i)}$ is the uuion of $k$ (i) disjoint irreducible subgraphs. Using the corollary to lemma 2 and noting, since $K^{(i)}$ is a proper subgraph of $K(i-1)$, that the inequality holds trivially when $k(i-1)=k(i)$, we have,

$$
\begin{aligned}
N(K(0))-N\left(K^{(1)}\right) & \geqslant k(1)-k^{(0)}+1 \\
N\left(K^{(1)}\right)-N\left(K^{(2)}\right) & \geqslant k^{(2)}-k^{(1)}+1 \\
& \cdot \\
& \cdot \\
N\left(K^{(t-2)}\right)-N\left(K^{(t-1)}\right) & \geqslant k(t-1)-k^{(t-2)}+1
\end{aligned}
$$

Adding these inequalities,

$$
N\left(K^{(0)}\right)-N(K(t-1))=k^{(t-1)}-k^{(0)}+t-1 \text {. }
$$

Since

$$
N(K(0))=N, N(K(t-1))=n, k(t-1)=n, k(0)=k,
$$

we have

$$
t \leq N-2 n+k+1
$$

Since $t_{k} \leqslant t$ the result follows.

If the matrix $A$ has no zeros, then $k=1$ and $N=n^{2}$ and hence $t_{k} \leq n^{2}-2 n+2$. In other cases, if the $k$ irreducible subgraphs have dimensions $n_{1}, n_{2}, \ldots, n_{k}$, $n_{1}+n_{2}+\ldots+n_{k}=n$, we have,

and

$$
N \leq n_{1}^{2}+n_{2}^{2}+\ldots+n_{k}^{2}
$$

$$
t_{k} \leqslant n_{1}^{2}+n_{2}^{2}+\ldots+n_{k}^{2}-2 n+k+1
$$




\section{REFERENCES}

1. G. Birkhoff, Tres observaciones sobre el álgebra lineal, Univ. Nac. Tucumán. Rev. Ser. A 5(1946), 147-150.

2. A.L. Dulmage and I. Halperin, On a theorem of Frobenius.Konig and $J$. von Neumann's game of Hide and Seek, Trans. Roy. Soc. Canada Sect. III 49 (1955), 23-29.

3. A.L. Dulmage and N.S. Mendelsohn, Coverings of bipartite graphs, Canad. J. Math. 10 (1958), 517-534.

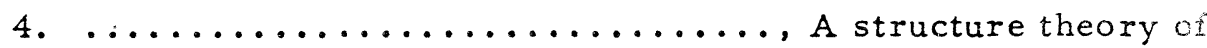
bipartite graphs of finite exterior dimension, Trans. Roy. Soc. Canada Sect. III 53 (1959), 1-13.

5. A.J. Hoffman and H.W. Wielandt, The variation of the spectrum of a normal matrix, Duke Math. J. 20 (1953), $37-39$.

6. M. Marcus and R. Ree, Diagonals of doubly stochastic matrices, Quart. J. Math. Oxford, Ser. (2), 10(1959), 296-302.

7. J. von Neumann, A certain zero-sum two-person game equivalent to the optimal assignment problem, Contributions to the Theory of Games, vol. II, Ann. of Math. Studies 28, $5-12$.

University of Manitoba 